\title{
Carrier Aggregation as a Repeated Game: Learning Algorithms for Efficient Convergence to a Nash Equilibrium
}

\author{
Hamed Ahmadi*, Irene Macaluso*, Luiz A. DaSilva*† \\ *CTVR Telecommunications Research Center, Trinity College Dublin, Ireland \\ ${ }^{\dagger}$ Department of Electrical and Computer Engineering, Virginia Tech, U.S.A. \\ Email: ahmadih,macalusi,dasilval@tcd.ie
}

\begin{abstract}
Carrier aggregation is a key feature of next generation wireless networks to deliver high-bandwidth links. This paper studies carrier aggregation for autonomous networks operating in shared spectrum. In our model, networks decide how many and which channels to aggregate in multiple frequency bands, hence extending the distributed channel allocation framework. Moreover, our model takes into the account physical layer issues, such as the out-of-channel interference in adjacent frequency channels and the cost associated with inter-band carrier aggregation. We propose learning algorithms that converge to Nash equilibria in a reasonable number of iterations under the assumption of incomplete and imperfect information.
\end{abstract}

Index Terms - Carrier aggregation, repeated game, learning, Nash equilibrium

\section{INTRODUCTION}

In this paper we address the distributed spectrum allocation problem for wireless networks that are able to simultaneously operate in multiple frequency bands. This is particularly important in light, for example, of the LTE-Advanced non-contiguous carrier aggregation (CA) techniques for $4 \mathrm{G}$ cellular networks, which allow mobile operators to aggregate spectrum from different bands to deliver high-bandwidth links [1]. Physical layer and implementation challenges of CA have been recently studied [2], [3]. In this work we study the dynamic aggregation of spectrum channels for multiple networks competing to access a common pool of spectrum resources.

Carrier aggregation, as currently envisaged, will involve the aggregation of static assignments of spectrum. However, recent works have explored its extension to dynamic contexts, where carriers are assigned to networks over shorter time frames on an as-needed basis [3]. In this case, it is possible to envision a coordination mechanism, either centralized or distributed, that manages the allocation of carriers to a number of networks. Alternatively, dynamic carrier allocation can be achieved in a distributed and uncoordinated manner: networks blindly interact in order to achieve a stable allocation of the available carriers. This paper adopts the latter approach. In our model,

This material is based upon works supported by the Science Foundation Ireland under Grants No. 10/CE/I1853 and 10/IN.1/I3007. each network, which initially exclusively occupies licensed carriers, has to decide how many and which carriers to aggregate from a common pool of spectrum resources. As we assume no exchange of information among the networks, we model the problem of dynamic carrier aggregation as a non-cooperative game and propose learning algorithms that converge to a pure Nash equilibrium (NE) within a reasonable number of iterations under the conditions of incomplete and imperfect information.

The problem of carrier aggregation has been studied in some recent works. In [4], the problem of distributed channel selection by frequency-agile radios is modeled as a game with incomplete information. The authors model the radios as learning automata and prove the convergence of the system to an NE. However, the convergence time of the proposed approach might hinder its practical application. A pricing game for spatial spectrum sharingbased carrier aggregation is proposed in [5]. The authors relate the pricing game to the power control game, and the aggregator's power optimization problem. Finally, they jointly optimize these problems for the scenario of two aggregating networks by using a Stackelberg game-based framework. In [6], the problem of dynamic inter-network carrier aggregation is studied, where mobile network operators decide whether to allow a portion of their spectrum to be aggregated by other operators for a limited time. The authors model the inter-network CA problem of two networks as a Bayesian game with incomplete information, and propose a distributed algorithm that approaches a neighborhood of the Bayesian NE of the system.

The problem posed in this work falls under the umbrella of managing coexistence among a group of networks operating in the same geographical area. The key challenge in managing coexistence is to avoid co-channel and adjacent channel interference. The first aspect has been widely studied in the context of distributed channel allocation [7], [8]. In this respect, our work extends the traditional channel selection problem by allowing each network to aggregate non-contiguous channels in multiple frequency bands (see Fig. 1).

Adjacent channel interference has usually been disregarded in previous works on distributed channel selection. Channels are commonly modeled as orthogonal, thus 


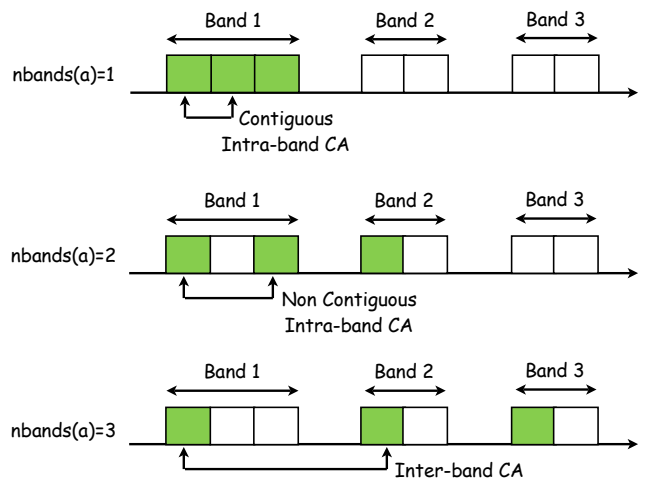

Fig. 1. Networks can transmit on multiple channels in the same frequency band (intra-band CA) or in multiple frequency bands (inter-band CA). The number of bands accessed by a network when selecting action $a$ is denoted by nbands $(a)$. In the three cases depicted in figure, the number of channels selected by the network is $|a|=3$. When $\operatorname{nbands}(a)=1$, the combination of channels corresponds to intra-band CA; when $\operatorname{nbands}(a)=2$, the combination of channels corresponds to a combination of intra and inter-band CA; when $\operatorname{nbands}(a)=3$, the combination of channels corresponds to interband CA.

relegating the problem of adjacent channel interference to the physical layer domain. In this paper, we adopt a different line. In fact, our model includes the effect of out-of-channel (OOC) interference in adjacent frequency channels. This way we achieve two objectives: we relax the assumption on channel orthogonality, and we are able to model the preference for contiguous channels aggregation. Furthermore, the model proposed in this paper also takes into account another important feature of CA. In fact, while allowing both intra- and inter-band CA, we assign a higher cost to the latter on account of the corresponding physical layer requirements.

\section{System MODEL}

Let us assume that $N$ wireless networks $(\mathcal{N}=$ $\{1,2, \ldots, N\})$ operate in the same geographical area where $B$ frequency bands $(\mathcal{B}=\{1,2, \ldots, B\})$ are available for dynamic usage. Each band has $K_{b}$ channels and the networks can simultaneously operate on multiple contiguous and non-contiguous channels. Although contiguous channel aggregation is preferable, non-contiguous channel aggregation is fully supported by all networks. In fact, in our model each network can decide how many and which channels to aggregate. Hence, if we denote by $M_{i}$ the maximum number of channels that network $i$ can access, the cardinality of each network's action space is:

$$
\left|\mathcal{A}_{i}\right|=\sum_{n=0}^{M_{i}}\left(\begin{array}{c}
M \\
n
\end{array}\right)
$$

where $M=\sum_{j=1}^{B} K_{j}$ is the total number of available channels. Each network is a player in the game that models the problem of dynamic CA. Action $a_{i}$ denotes the set of channels selected by player $i$; nbands $\left(a_{i}\right)$ is the number of bands that node $i$ accesses when selecting action $a_{i}$ (nbands $\left.\left(a_{i}\right) \leq\left|a_{i}\right|\right) ; N_{B_{i}}$ is the maximum number of bands that player $i$ can simultaneously use. Fig. 1 depicts a scenario where $B=3$ bands are considered: depending on the combination of channels selected by the network the resulting scheme is intra-band $\mathrm{CA}$, inter-band $\mathrm{CA}$, or a combination of the two.

The model we propose assumes that the networks indirectly interact by causing interference to each other. Their objective is to achieve a stable allocation of the spectrum resources. In general, we can define the interference measured by network $i$ on channel $c_{k}$ as result of the transmission of other networks as:

$$
\sum_{j=1, j \neq i}^{N} \operatorname{emission}\left(a_{j}, c_{k}\right),
$$

where emission $\left(a_{j}, c_{k}\right)$ is the interference in channel $c_{k}$ caused by network $j$ transmitting on the set of channels $a_{j}$. If channels are modeled as orthogonal, emission $\left(a_{j}, c_{k}\right)$ is null if $\forall c_{l} \in a_{j}, f_{l}-f_{k} \neq 0$, where $f_{l}$ and $f_{k}$ denote the center frequency of channel $c_{l}$ and $c_{k}$, respectively. In our model, we relax the assumption on channel orthogonality. Hence, the function emission $\left(a_{j}, c_{k}\right)$ is null if $\forall c_{l} \in a_{j},\left|f_{l}-f_{k}\right|>\bar{f}$, where $\bar{f}$ is the maximum center frequency distance beyond which spectral emissions generated by other networks can be neglected. We approximate the function emission $\left(a_{j}, c_{k}\right)$ by assuming that the power spectrum of the actual waveform transmitted by each radio equipment has been tailored to comply with a predefined spectral mask.

The payoff function combines two different aspects of the problem: the interference experienced as result of the transmissions of neighboring networks and the cost incurred for inter-band CA. The last aspect cannot be neglected, as inter-band CA poses serious challenges on the radio front-end and requires increased computational resources. For example, CA with non-contiguous component carriers requires separate fast Fourier transforms [9]. The preference for contiguous channel assignments is implicitly modeled in the payoff function by taking into account the OOC emissions in the computation of the interference. We define the payoff of player $i$ as:

$$
r_{i}(\mathbf{a})= \begin{cases}\frac{1}{M_{i}} \sum_{c_{i} \in a_{i}}\left(1-\frac{\gamma_{c_{i}}}{\overline{\gamma_{i}}}\right)-\frac{\left(\operatorname{nbands}\left(a_{i}\right)-1\right) \delta}{N_{B_{i}}} & \text { if } a_{i} \neq \emptyset \\ 0 & \text { if } a_{i}=\emptyset\end{cases}
$$

where $\gamma_{c_{i}}$ is the interference measured in channel $c_{i}$ and normalized in the range $[0,1], \bar{\gamma}_{i}$ is the maximum interference that player $i$ can tolerate on a channel (also normalized in $[0,1]$ ), and $\delta$ is a parameter that each network can tune to reflect its degree of flexibility in performing CA.

In order to understand the effect of the cost associated with inter-band $\mathrm{CA}$, let us consider action $a_{i}$ and its associated payoff $r_{i}\left(a_{i}, a_{-i}\right)$. We analyze the effect on the received payoff if player $i$ unilaterally changes its strategy and selects action $a_{i}^{\prime}$ corresponding to transmitting on one additional channel, denoted by $c_{i}^{\prime}$, with respect to $a_{i}$. To study the payoff difference $\Delta r^{\prime}=r_{i}\left(a_{i}^{\prime}, a_{-i}\right)-r_{i}\left(a_{i}, a_{-i}\right)$, 


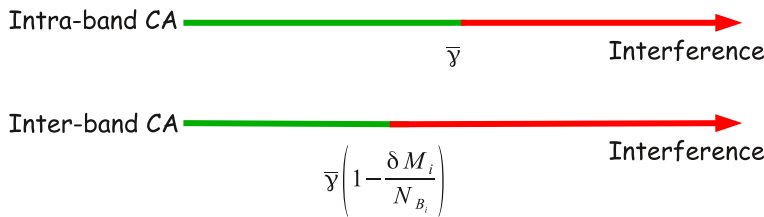

Fig. 2. Interference and cost of inter-band CA. The green intervals represent the ranges of interference values for which it is beneficial to transmit on a channel. Transmitting on a channel in an extra band, i.e. increasing the number of bands accessed by the network, has a cost which is modeled by restricting the range of interference values corresponding to an increment of the received payoff. Each network can tune the parameter $\delta$ to reflect its degree of flexibility in performing $\mathrm{CA}$.

we have to consider two separate cases:

- Intra-band CA $\left(\operatorname{nbands}\left(a_{i}^{\prime}\right)=\operatorname{nbands}\left(a_{i}\right)\right)$ : $\Delta r^{\prime}=\frac{1}{M_{i}}\left(1-\frac{\gamma_{c_{i}^{\prime}}}{\overline{\gamma_{i}}}\right)$.

- Inter-band CA (nbands $\left.\left(a_{i}^{\prime}\right)=\operatorname{nbands}\left(a_{i}\right)+1\right)$ : $\Delta r^{\prime}=\frac{1}{M_{i}}\left(1-\frac{\gamma_{c_{i}^{\prime}}}{\bar{\gamma}_{i}}\right)-\frac{\delta}{N_{B_{i}}}$.

In the case of intra-band $\mathrm{CA}$, it is beneficial for player $i$ to transmit on the additional channel $c_{i}^{\prime}\left(\Delta r^{\prime}>0\right)$ if the interference in $c_{i}^{\prime}$ is less than the maximum interference the radio can tolerate. In the case of inter-band $\mathrm{CA}$, it is beneficial to add the additional channel $c_{i}^{\prime}$ if the interference in $c_{i}^{\prime}$ is less than the maximum interference the radio can tolerate, decreased by $\frac{\delta M_{i} \overline{\gamma_{i}}}{N_{B_{i}}}$. Analogous results can be obtained by analyzing the effect on the payoff difference when a network unilaterally decides to remove one channel from the set of channels selected for transmission.

In summary, the effect of the cost associated with interband $\mathrm{CA}$ is to restrict the range of interference values for which it is beneficial for a network to transmit on a channel. Figure 2 represents this concept diagrammatically.

As a result of the above discussion, we can formulate the distributed CA problem as a game denoted by $\mathcal{G}=$ $(\mathcal{N}, \mathcal{A}, r)$. In this game, $\mathcal{N}$ is the set of players, which are the radio networks, $\mathcal{A}=\times \mathcal{A}_{i}$ is the set of their possible actions, and $r$ is the payoff function in (3).

\section{NASH EQUILIBRIA}

In this section we show that the $\mathrm{CA}$ game $\mathcal{G}$ has at least one Nash equilibrium in pure strategies, and in the next section we present methods that lead the game to converge to an NE.

Any pure strategy corresponding to a collision (i.e. two or more networks transmitting on overlapping sets of channels) is not a Nash Equilibrium. According to the payoff defined above, if player $i$ transmits on a set of channels and a subset of those channels are occupied by other networks, it is beneficial for player $i$ to unilaterally decide to transmit only on the collision-free channels. Knowing this, in Proposition 1, we prove the existence of a pure NE for our proposed CA game.
Proposition 1: At least one Nash equilibrium in pure strategies exists in CA game $\mathcal{G}$.

Proof: Initially we assume that all the channels in all bands are free. We randomly select a player from the set of waiting players $(i \in \mathcal{N})$ and let her select the action that maximizes her payoff knowing the actions of all other players $\left(\bar{a}_{i}=\operatorname{argmax}_{a_{i}} r_{i}\left(a_{i}, \bar{a}_{-i}\right), a_{i} \in \mathcal{A}_{i}\right)$. Then, we remove the player from the set of waiting players. We repeatedly perform the aforementioned actions until either all the channels are assigned to players or the set of waiting players is empty. At this point none of the players can improve her payoff by unilaterally changing her action, which indicates that it is an NE.

The payoff function and, more specifically, the cost associated with inter-band CA play an important role in defining the behavior of the players and characterizing the NE. If the cost associated with $\mathrm{CA}$ is low enough that for a network it is convenient to perform CA even when only one channel in the extra band can be added, then a pure NE corresponds to players aggregating a total number of channels equal to $\min \left(\sum_{j=1}^{N} M_{j}, M\right)$. In other words, for a network it is always convenient to add a channel that exhibits an interference level less than $\overline{\gamma_{i}}$ until the maximum number of channels is reached. If the cost of $\mathrm{CA}$ is such that for a network it is not beneficial to aggregate a single channel in an extra band, it is possible that some channels will remain unoccupied, even though some networks have not reached the maximum number of channels they can transmit on.

\section{LEARning the NASh Equilibria}

In this section we present and compare the performance and requirements of learning algorithms that lead the players to a pure NE. The payoff defined in (3) allows a fully distributed solution to the CA game, as we will see in the remainder of this section. At each stage $k$, each player $i$ chooses an action according to one of the learning algorithms described below and observes a response from the environment. The response provided to the player (transmitter) by its receivers is the estimated level of interference in the channels selected for transmission by the player in stage $k$. This feedback is used by the player to compute the payoff of the action selected at time $k$ (received payoff ). In some cases, the feedback also includes the estimated level of interference in all the available channels. This information is used to compute the payoffs that the player would have received, had she selected a different combination of channels at time $k$ (hypothetical payoff). This additional information can significantly reduce the convergence time to an NE.

\section{A. Interactive trial and error learning (ITEL)}

The ITEL algorithm, proposed in [10], is a simple but effective learning algorithm. Players do not need any information about other players and the payoff of their own other possible actions. In [10], it is proven that ITEL converges to a pure NE with high probability. 
ITEL can be denoted by a tuple $z_{i}=\left(m_{i}, \bar{a}_{i}, \bar{r}_{i}\right)$ for each player, where $\bar{a}_{i}$ and $\bar{r}_{i}$ denote the benchmark action and benchmark utility (payoff), respectively. In the ITEL tuple, $m_{i}$ denotes the player's mood, which can be either content, discontent, hopeful, or watchful. The current action and current utility are denoted by $a_{i}$ and $r_{i}$, respectively.

A content player plays the benchmark action with probability $p_{\bar{a}_{i}}=1-\epsilon$ and tries a random action from its action set $\left(\mathcal{A}_{i}\right)$ with probability $p_{a_{i} \neq \bar{a}_{i}}=\epsilon$. In other words, $\epsilon$ is the exploration rate, which should be very small.

If a content player tries a new action and receives a higher payoff $\left(\bar{r}_{i}<r_{i}\right)$, it will update its benchmark action and benchmark utility to the new action and the new utility, respectively. Otherwise, it will maintain its benchmark action for the next time slot. It is possible that a content player plays its benchmark action and receives a higher or lower payoff than its benchmark payoff due to the actions of other players. Playing the benchmark action, if the received utility of a content player is higher than the benchmark utility, she maintains the benchmark utility and benchmark action, but changes her mood to hopeful. On the other hand, playing the benchmark action, if the received utility of a content player is lower than the benchmark utility, she maintains the benchmark utility and benchmark action, but changes her mood to watchful.

A hopeful player always plays the benchmark action, and if she receives the same utility as the benchmark utility she will become content, maintaining her benchmark action and utility. However, if she receives a higher utility than her benchmark utility $\left(r_{i}>\bar{r}_{i}\right)$, then she will update her benchmark utility to the new utility $\left(\bar{r}_{i}=r_{i}\right)$, and become content. It is possible that the hopeful player receives a lower utility than her benchmark utility. In this case, the player maintains her benchmark action and utility, but changes her mood to watchful.

A watchful player always plays the benchmark action, and if she receives the same utility as the benchmark utility she will return to the content mood, maintaining her benchmark action and utility. Otherwise, if she receives a higher utility than her benchmark utility $\left(r_{i}>\bar{r}_{i}\right)$, she will update her mood to hopeful. On the other hand, if she receives a utility lower than her benchmark utility $\left(r_{i}<\bar{r}_{i}\right)$, she will update her mood to discontent. This means a content player will become discontent only if, at two consecutive stages, she receives a lower utility than the benchmark utility, while playing the benchmark action.

A discontent player plays an action $\left(a_{i}\right)$ which is uniformly drawn from her action set $\left(\mathcal{A}_{i}\right)$. She becomes content with probability $\phi\left(r_{i}(\mathbf{a}), \bar{r}_{i}\right)$ where $\phi$ is monotone increasing in $r_{i}$ and monotone decreasing in the benchmark $\bar{r}_{i}$. Moreover, $\phi$ is bounded away from 0 and 1 $\left(\theta \leq \phi\left(r_{i}, \bar{r}_{i}\right) \leq 1-\theta\right.$, for some $\left.0<\theta<1\right)$. If the player becomes content the selected action and its utility will become the benchmarks.

The algorithm starts from a random action for each player and continues by updating the action, utility and mood of the players according to the aforementioned rules.
TABLE I

UPDATING THE $z_{i}$ BASED ON THE ACTIONS AND OBSERVATIONS.

\begin{tabular}{|c|c|c|c|}
\hline$z_{i}=\left(m_{i}, \bar{a}_{i}, \bar{r}_{i}\right)_{t}$ & Action & Utility & $z_{i}=\left(m_{i}, \bar{a}_{i}, \bar{r}_{i}\right)_{t+1}$ \\
\hline \multirow{4}{*}{$z_{i}=\left(c, \bar{a}_{i}, \bar{r}_{i}\right)$} & $a_{i}=a_{i}^{*}$ & 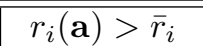 & $\overline{\bar{z} z_{i}\left(c, a_{i}, r_{i}(\mathbf{a})\right)}$ \\
\hline & $a_{i}=\bar{a}_{i}$ & $r_{i}(\mathbf{a})<\bar{r}_{i}$ & $z_{i}\left(w, \bar{a}_{i}, \bar{r}_{i}\right)$ \\
\hline & $a_{i}=\bar{a}_{i}$ & $r_{i}(\mathbf{a})>\bar{r}_{i}$ & $z_{i}\left(h, \bar{a}_{i}, \bar{r}_{i}\right)$ \\
\hline & $a_{i}=\bar{a}_{i}$ & $r_{i}(\mathbf{a})=\bar{r}_{i}$ & $z_{i}\left(c, \bar{a}_{i}, \bar{r}_{i}\right)$ \\
\hline \multirow{3}{*}{$z_{i}=\left(h, \bar{a}_{i}, \bar{r}_{i}\right)$} & & $r_{i}(\mathbf{a})<\bar{r}_{i}$ & $z_{i}\left(w, \bar{a}_{i}, \bar{r}_{i}\right)$ \\
\hline & $a_{i}=\bar{a}_{i}$ & $r_{i}(\mathbf{a})=\bar{r}_{i}$ & $z_{i}\left(c, \bar{a}_{i}, \bar{r}_{i}\right)$ \\
\hline & & $r_{i}(\mathbf{a})>\bar{r}_{i}$ & $z_{i}\left(c, \bar{a}_{i}, r_{i}(\mathbf{a})\right)$ \\
\hline \multirow{3}{*}{$z_{i}=\left(w, \bar{a}_{i}, \bar{r}_{i}\right)$} & & $r_{i}(\mathbf{a})<\bar{r}_{i}$ & $z_{i}\left(d, \bar{a}_{i}, \bar{r}_{i}\right)$ \\
\hline & $a_{i}=\bar{a}_{i}$ & $r_{i}(\mathbf{a})=\bar{r}_{i}$ & $z_{i}\left(c, \bar{a}_{i}, \bar{r}_{i}\right)$ \\
\hline & & $r_{i}(\mathbf{a})>\bar{r}_{i}$ & $z_{i}\left(h, \bar{a}_{i}, r_{i}(\mathbf{a})\right)$ \\
\hline \multirow{3}{*}{$z_{i}=\left(d, \bar{a}_{i}, \bar{r}_{i}\right)$} & \multirow{3}{*}{$a_{i}=a_{i}^{*}$} & $\begin{array}{l}\text { with prob } \\
\phi\left(r_{i}(\mathbf{a}), \bar{r}_{i}\right)\end{array}$ & $z_{i}\left(c, a_{i}, r_{i}(\mathbf{a})\right)$ \\
\hline & & with prob & . $(0$ \\
\hline & & $1-\phi\left(r_{i}(\mathbf{a}), \bar{r}_{i}\right)$ & $z_{i}$ \\
\hline
\end{tabular}

\section{B. ITEL Best Action (ITEL-BA)}

ITEL is designed for scenarios where the players can only observe the results of their own actions. As previously observed, ITEL converges to a pure NE. In [11], the authors applied this result to the discrete power allocation problem, and they observed that the number of iterations required to be close to a Nash equilibrium depends on $\epsilon$ and on the structure of the observed payoffs. The algorithm we present in this section is based on the observation that it is possible to significantly reduce the convergence time to an NE, if the players are able to gather additional information. Our proposed adaptation mechanism, which we call ITEL-BA, focuses on the selection of a proper action when a player experiments with new actions. We assume that the networks are able to sense and estimate the level of interference on all the available channels. ITEL-BA exploits this additional information by selecting the action that maximizes the achievable utility of the network, based on the hypothetical payoffs estimate. The learning algorithm is the same as ITEL, but for the action selection mechanism during the exploration stage. In ITEL-BA, when a player experiments with new actions, either in content or discontent mood, she will select her action according to:

$$
a_{i}^{*}=\operatorname{argmax}_{i}\left(a_{i}^{\prime}, \bar{a}_{-i}\right) \quad \forall a_{i}^{\prime} \in \mathcal{A}_{i}
$$

Table I illustrates how the mood and benchmarks will be updated for the next game according to the selected action and observation of the achieved utility.

ITEL-BA converges to a pure NE. The proof follows the fact that ITEL converges to a pure NE for any (randomly) selected action in $\mathcal{A}_{i}$ [10], and the best action is an action in $\mathcal{A}_{i}$ which can be selected. Thus, to prove that ITELBA also converges to a NE based on the proof of ITEL we need to show that restricting the action selection to the best action does not affect the convergence conditions.

In ITEL-BA (and ITEL), a player tries a new action only when she is in a discontent mood and/or with 


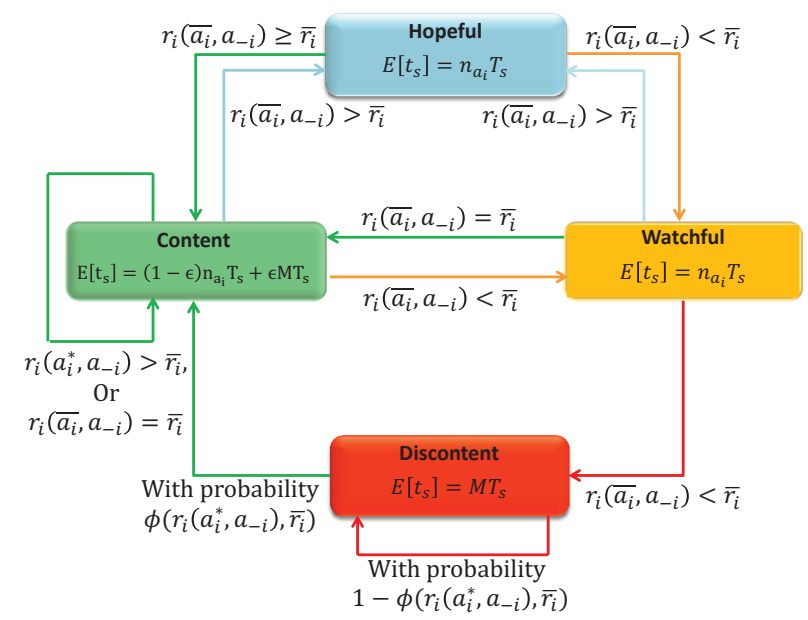

Fig. 3. State transitions in ITEL-BA, and the expected sensing time at each state. The required time for sensing one channel is $T_{s}$.

probability of $\epsilon$ when she is content. In the case of a discontent player, selecting the best action increases $\phi\left(u_{i}(\mathbf{a}), \bar{u}_{i}\right)$, which leads to a higher probability of leaving the discontent mood. According to the ITEL proof in [10], in an NE state all players are content. Thus, selecting the best possible action accelerates reaching a state where all players are content, and it does not violate the convergence conditions. For a content player, there is a possibility to experiment with a new action. In ITELBA, the player selects the action which maximizes her utility (best action). If the best action is the benchmark action, she will remain content and keep the benchmark action and utility, but if playing the best action increases the utility, the player will stay content and update her benchmarks (benchmark action and benchmark utility). Certainly, there are situations where all the players are content but the game is not in an NE. In such situations, at least one player can improve her utility by changing her action. If this player tries the best action she will stay content with her best current utility. However, she may cause some other players to become watchful and then discontent. The game will be played again and as shown in [10] it will reach a state such that all the players are content and cannot improve their utility by changing their action. Therefore, playing the best action when a player is content does not violate the convergence conditions.

The above discussions show that convergence of ITEL$\mathrm{BA}$ to a NE can be proved using the same proof as ITEL provided in [10].

It is important to notice that applying ITEL-BA is only possible when the networks are able to estimate the interference on all the available channels, hence to compute the payoff of all their possible actions. In this problem the available information is still incomplete because we assume that each network cannot identify which other network is transmitting on a specific channel and causing interference. The networks are only able to measure the aggregate level of interference on the channels.

It is also important to remark that a network is required to compute the hypothetical payoffs, i.e. to sense all the available channels, only when experimenting with new actions either in content or discontent mood. In all other cases, only the interference in the channels selected for transmission is required for the eventual update of the mood and the benchmark utility. Fig. 3 shows the expected sensing time $E\left[t_{s}\right]$ for all the states, assuming that the sensing time for a single channel is $T_{s}$.

\section{ITEL Best Action with Imperfect Information (ITEL- $B A W I I)$}

ITEL-BA assumes perfect interference estimation. Although recent achievements in sensing technologies have significantly improved the interference estimation accuracy, the assumption of perfect interference estimation is too optimistic, especially in the case of multichannel spectrum sensing. In addition, some networks may not be able to sense all the channels, especially when the number of channels to monitor is large. In ITEL-BAWII, we remove this assumption and consider noisy and imperfect sensing information for the channels the network is not transmitting on.

To counteract the effects of noisy observations on the convergence time, each network keeps an estimate of the payoff over the set of actions $\left(\hat{r}_{i}(k)\right)$. At each stage $k$, each player $i$ chooses an action and receives a feedback that includes the estimated level of interference in all the available channels. Each player computes the received and hypothetical payoffs and then updates $\hat{r}_{i}(k)$ using an $n$ sample weighted moving average. By assigning greater weight to the most recent payoffs estimates, each player is able to react more quickly to the dynamics of the other players. In ITEL-BAWII, when a player experiments with new actions either in content or discontent mood, she will select the action that maximizes the average estimated payoff $\hat{r}_{i}(k)$. ITEL-BAWII significantly reduces the convergence time to an NE in the presence of noisy observations by averaging the payoffs estimate over a fixed time frame. This comes with an increased cost in terms of the additional resources required to monitor the interference level in all the available channels. In fact, the expected sensing time is $T_{s} M$ for all the states.

\section{Simulation Results AND Discussion}

As discussed in Section II, in this work we take into account the OOC emissions in adjacent frequency channels, when computing the interference caused to neighboring networks. To do this, we approximate the emissions of a transmitter with the spectrum emission mask (SEM) defined for user equipment in LTE [12]. This represents a worst case scenario: as all the transmitters will have to comply with the limits defined by the SEM, the actual emissions will at most equal the SEM. We chose to use the SEM defined in LTE because of the variable-bandwidth definition, which is also a characteristic of our model. As the SEM is defined for OOC emissions relative to the in-channel power, without loss of generality the inband 


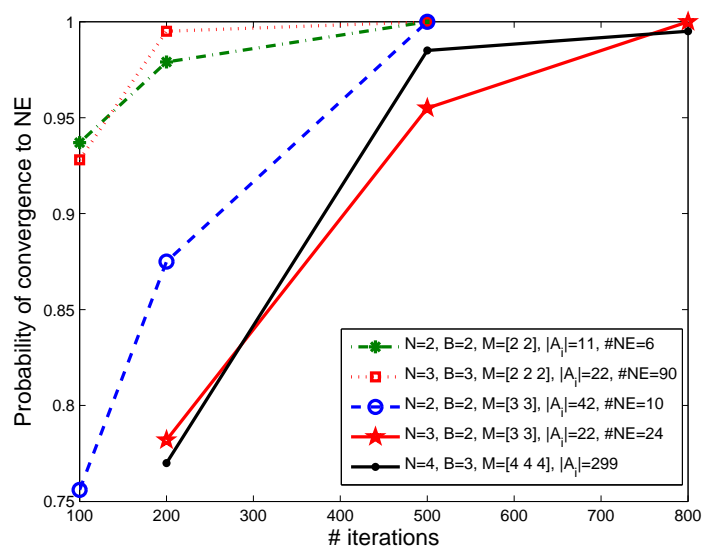

Fig. 4. Convergence probability of ITEL-BA to an NE for different scenarios. $M$ shows the number of channels in each band, and $\left|A_{i}\right|$ shows the cardinality of the action set of network $i$.

power limit is normalized to $0 \mathrm{dBm}$ in all the experiments. Moreover, in all our simulations the channel bandwidth is $5 \mathrm{MHz}$ and the exploration rate of the learning algorithms is $\epsilon=0.01$. The parameter $\delta$ is chosen so that for a network it is beneficial to aggregate a single channel in an additional band if at most one of the adjacent channels is occupied by another network.

We tested ITEL for a simple scenario where $N=2$ networks compete to access a total of 4 channels distributed across $B=2$ bands. Even for this simple scenario we need $10^{5}$ iterations to obtain a probability of convergence to an $\mathrm{NE}$ of 0.90 . In Fig. 4, we present and compare the probability of convergence to an NE for scenarios where different numbers of ITEL-BA-adopting networks compete for aggregation of channels in different numbers of bands. For the same scenario discussed above for ITEL, only $10^{2}$ iterations are necessary for ITEL-BA to obtain a probability of convergence of 0.90 (green line in Fig. 4). The three order of magnitude improvement in the convergence time is gained at the cost of minor additional sensing requirements. We also analyze the impact of the cardinality of the action space (which is a function of the number of available channels and desired channels), the number of bands, and the distribution of the available channels on those bands, on the probability of convergence to an NE. These elements, along with the cost associated with inter-band CA, affect the number of pure NE of the CA game. It is important to note that two scenarios characterized by the same number of networks, bands and total number of available channels, exhibit a different number of $\mathrm{NE}$ based on the distribution of available channels. As we can observe in Fig. 4, ITEL$\mathrm{BA}$ requires more iterations to converge to an $\mathrm{NE}$ with a high probability as the number of networks and/or the cardinality of each player's action space increases. These elements being equal, a larger number of $\mathrm{NE}$ corresponds to a shorter convergence time.

Fig. 5 clearly illustrates how noisy and imperfect observations can degrade the performance of ITEL-BA. In

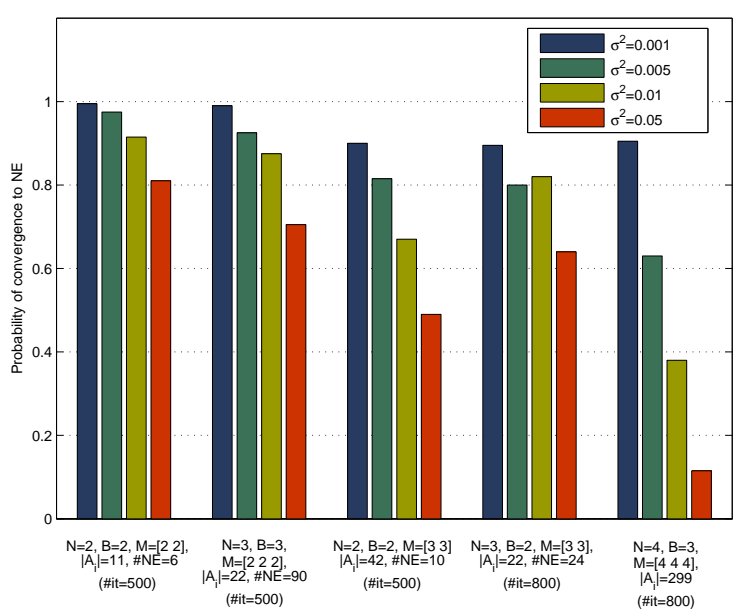

Fig. 5. Convergence probability of ITEL-BA when the observations are not perfect. The imperfection of the observations is modeled as additive zero mean Gaussian noise with standard deviation of $\sigma^{2}$.

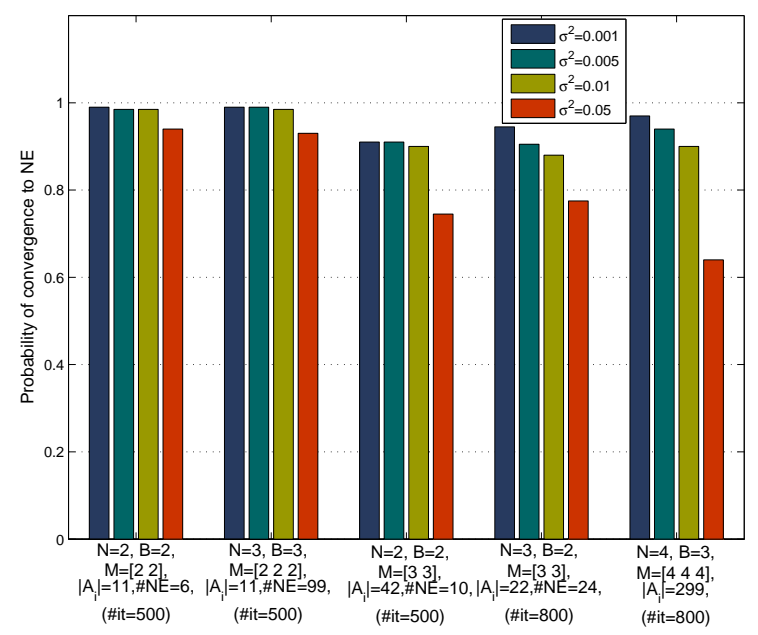

Fig. 6. Convergence probability of ITEL-BAWII when the observations are not perfect. The imperfection of the observations is modeled as additive zero mean Gaussian noise with standard deviation of $\sigma^{2}$.

this scenario, to generate imperfect observations we added noise drawn from a $\mathcal{N}\left(0, \sigma^{2}\right)$ distribution to the interference on the channels that the player is not accessing. In Fig. 5, we observe that when the standard deviation of the noise is low $\left(10^{-3}\right)$ the performance of ITEL-BA is only slightly reduced; as the noise level increases, the performance of ITEL-BA is significantly degraded. The effect of imperfect observations increases with the number of networks competing for the channels and/or with the cardinality of each player's action space. These elements being equal, a larger number of $\mathrm{NE}$ reduces the impact of imperfect observations on the performance of ITELBA in terms of convergence probability. Fig. 6 shows the probability that the same carrier aggregation games converge to an NE when the players adopted ITEL-BAWII. As shown in the figure, ITEL-BAWII significantly increases the probability that CA games converge to a pure NE even for the scenarios characterized by very noisy observations. 
By comparing Fig. 5 with Fig. 6, we can conclude that ITEL-BAWII significantly outperforms ITEL-BA when the observations are noisy and imperfect. For example, in the case of $N=4$ networks, even tripling the maximum number of iterations for ITEL-BA only achieves a probability of convergence to an NE of 0.31 . However, it is important to keep in mind that networks that adopt ITELBA need to sense the interference level of other channels and compute the utility of their other possible actions only when they are experimenting with actions other than their benchmark action. Moreover, ITEL-BA does not need to keep a history of observations. Therefore, due to the lower complexity and high performance of ITEL-BA, it is preferred to ITEL-BAWII when high quality and perfect observation is possible.

To compare ITEL-BA performance with the performance of another learning algorithm, we simulated a simultaneous best response dynamics (BRD) strategy. Simultaneous BRD assumes that at each time slot every player plays the action that maximizes its own payoff given the actions of all other players at the previous time slot [13]. Our simulation results, considering the scenarios described in Fig. 4, show that simultaneous BRD does not have an acceptable performance for the proposed CA game. For a simple scenario where $N=2$ networks compete to access a total of 4 channels distributed in $B=2$ bands, the probability of convergence to an NE of $\mathrm{BRD}$ is 0.22 after $10^{5}$ iterations. The convergence rates deteriorate for more complicated scenarios.

Sequential BRD is another variation of BRD, where at each time only one player can change its action and select the action that maximizes its payoff. On the one hand, it is not difficult to show that the CA game can converge to an $\mathrm{NE}$ using sequential BRD in few iterations. On the other hand, it is necessary to envision a form of coordination to prevent more than one player from changing their action at the same time. Since in this work we analyzed a distributed and uncoordinated solution, we cannot apply sequential $\mathrm{BRD}$ to the presented $\mathrm{CA}$ game.

\section{Conclusions}

In this work we modeled the CA problem of autonomous networks operating in shared spectrum as a repeated game. We proposed learning algorithms that efficiently converge to an NE without the need for complete or even perfect information. Our results show that the algorithm, which effectively converges to an NE with incomplete information (ITEL-BA), is not efficient in the case of imperfect information. Our algorithm that effectively deals with imperfect and incomplete information (ITELBAWII) requires additional sensing and computational resources.

Unlike previous works on distributed channel selection, which model channels as orthogonal, the model proposed in this paper takes into account adjacent channel interference in the computation of the payoff. Adjacent channel interference is particularly important when a network can aggregate multiple channels. In fact, by relaxing the assumption on channel orthogonality, the preference for contiguous channels is implicitly expressed in any payoff function that decreases when the interference increases.

\section{REFERENCES}

[1] G. Yuan, X. Zhang, W. Wang, and Y. Yang, "Carrier Aggregation for LTE-Advanced Mobile Communication Systems," IEEE Commun. Mag., vol. 48, pp. 88-93, 2010.

[2] D. Bai, C. Park, J. Lee, H. Nguyen, J. Singh, A. Gupta, Z. Pi, T. Kim, C. Lim, M. Kim, and I. Kang, "LTE-advanced modem design: challenges and perspectives," IEEE Commun. Mag., vol. 50, no. 2, pp. 178-186, 2012.

[3] K. Pedersen, F. Frederiksen, C. Rosa, H. Nguyen, L. Garcia, and Y. Wang, "Carrier aggregation for LTE-advanced: functionality and performance aspects," IEEE Commun. Mag., vol. 49, no. 6, pp. 89-95, 2011.

[4] I. Macaluso, L. DaSilva, and L. Doyle, "Learning Nash Equilibria in Distributed Channel Selection for Frequency-agile Radios," in Workshop on Artificial Intelligence for Telecommunications and Sensor Networks, 2012.

[5] Y. Xiao, T. Forde, I. Macaluso, L. DaSilva, and L. Doyle, "Spatial spectrum sharing-based carrier aggregation for heterogeneous networks," in IEEE Global Telecommunications Conference (GLOBECOM 2012), pp. 2615-2620, Dec. 2012.

[6] Y. Xiao, C. Yuen, P. Di Francesco, and L. DaSilva, "Dynamic Spectrum Scheduling for Carrier Aggregation: A Game Theoretic Approach," in IEEE ICC'13.

[7] C. Wu, K. Chowdhury, M. Di Felice, and W. Meleis, "Spectrum management of cognitive radio using multi-agent reinforcement learning," in Proceedings of the 9th International Conference on Autonomous Agents and Multiagent Systems: Industry track, pp. 1705-1712, International Foundation for Autonomous Agents and Multiagent Systems, 2010.

[8] D. Leith and P. Clifford, "Convergence of distributed learning algorithms for optimal wireless channel allocation," in 45th IEEE Conf. Decision and Control, pp. 2980-2985, IEEE, 2006.

[9] D. Bai, C. Park, J. Lee, H. Nguyen, J. Singh, A. Gupta, Z. Pi, T. Kim, C. Lim, M. Kim, et al., "LTE-advanced modem design: challenges and perspectives," Communications Magazine, IEEE, vol. 50, no. 2, pp. 178-186, 2012.

[10] H. Young, "Learning by trial and error," Games and economic behavior, vol. 65, no. 2, pp. 626-643, 2009.

[11] S. Lasaulce and H. Tembine, Game Theory and Learning for Wireless Networks: Fundamentals and Applications. Academic Press, 2011.

[12] 3GPP Technical Specification 26.101, "LTE; Evolved Universal Terrestrial Radio Access (E-UTRA); User Equipment (UE) radio transmission and reception," www.3gpp.org.

[13] L. Rose, S. Lasaulce, S. Perlaza, and M. Debbah, "Learning equilibria with partial information in decentralized wireless networks," IEEE Commun. Mag., vol. 49, no. 8, pp. 136-142, 2011 . 